\title{
Office building participation in demand response programs supported by intelligent lighting management
}

\author{
Mahsa Khorram, Omid Abrishambaf, Pedro Faria* 'D and Zita Vale
}

\author{
* Correspondence: \\ pnsfaria@gmail.com \\ GECAD - Research Group on \\ Intelligent Engineering and \\ Computing for Advanced \\ Innovation and Development, \\ Institute of Engineering - \\ Polytechnic of Porto (ISEP/IPP), Rua \\ Dr. António Bernardino de Almeida, \\ 431, 4200-072 Porto, Portugal
}

\begin{abstract}
According to importance of demand response programs in smart grids and microgrids, many efforts have been made to change the consumption patterns of the users, and the use of renewable resources has also increased. Significant part of energy consumption belongs to buildings such as residential, commercial, and office buildings. Many buildings are equipping with components that can be used for the participation in demand response programs. The SCADA system plays a key role in this context, which enables the building operator to have control and monitor the consumption and generation. This paper presents a real implementation of an optimization based SCADA system, which employs several controlling and monitoring methods in order to manage the consumption and generation of the building for decision support and participating in demand response events. Since the air conditioning devices are suitable controllable appliances for direct load control demand response, and lighting system as flexible loads for reduction and curtailment, they can play a key role in the scope of demand response programs. In this system, several real controller components manage the consumption of lighting system and air conditioning of the building based on an optimization model. In the case study of the paper, the SCADA system is considered as a player of an aggregation model, which is considered as demand response managing entity, and its performance during demand response events will be surveyed. The obtained results show that with adequate small reduction in the lighting system and air conditioning devices, the electricity customers are able to actively participate in the electricity markets using demand response programs and also for internal efficient use of electricity.
\end{abstract}

Keywords: Demand response, Optimization, SCADA, Aggregation

\section{Background}

The idea of Demand Response (DR) is a fact in the current distribution networks, and is referred to modification of consumption pattern in end-users for responding to the electricity price variations or due to the technical or economic reasons (Ruelens et al. 2017). There are two main types of DR programs: incentive-based, and price-based (Faria and Vale 2011). The incentive-based DR is related to a program in which the customers are paid with the fixed or time varying incentive, provided by the grid operator or other DR provider (Fotouhi Ghazvini et al. 2017). The price-based DR programs are referred to the changes in the consumption of the customers when

(c) The Author(s). 2018 Open Access This article is distributed under the terms of the Creative Commons Attribution 4.0 International License (http://creativecommons.org/licenses/by/4.0/), which permits unrestricted use, distribution, and reproduction in any medium, provided you give appropriate credit to the original author(s) and the source, provide a link to the Creative Commons license, and indicate if changes were made. 
facing electricity price variations (Lujano-Rojas et al. 2017). In this concept, the end-users tend to participate in such programs in order to reduce their electricity bills by shifting their high consumption appliances to the off-peak hours, or reduce their high consumption loads in peak hours (Hu et al. 2017). Beside the DR programs, the implementation of Distributed Renewable Energy Resources (DRERs) in demand side, is a key role in smartgrids (Abrishambaf et al. 2017). This means that the consumers will not only be able to supply the local demand through the onsite generation resources, but also, they can sell energy to the network when there is generation surplus (Abrishambaf et al. 2016).

Currently, most of the implemented DR programs are driven to the large-scale resources. However, a huge potential of DR participation is available in small-scale resources. This means that small consumers, such as residential or commercial buildings, cannot participate in the DR programs individually (Kwon et al. 2017). In order to overcome this issue, several concepts have been proposed. Virtual Power Player (VPP) (Faria et al. 2013), and Curtailment Service Provider (CSP) (Wang et al. 2017) are two concepts that can overcome the mentioned barrier. The DR aggregation, made by entities such as VPP, CSP, or other type of aggregator is a solution for the participation of large amounts of consumers in DR programs and other electricity market products (Parvania et al. 2013). These concepts can be defined, as in (Faria et al. 2016), as an aggregation network that aggregate small and medium scale consumers and prosumers, namely to participate in the market as one.

In fact, an effective implementation of DR, namely at small consumers and buildings level requires adequate means for the communication, measurement, and validation concerning the actual participation of the consumers in the DR events, for incentive-based DR programs. In this way, the consumer should be able to receive the information regarding the DR events from DR managing entities, and execute them (Paterakis et al. 2017). Looking at commerce and services buildings, in last decades, Supervisory Control And Data Acquisition (SCADA) system has a key role for monitoring and controlling the centralized management infrastructure (Hasan and Mouftah 2016). With the support of a SCADA system, lighting system and Air Conditioning (AC) devices can play an important role for participating in DR programs. The lights are considered as flexible loads for reduction and curtailment (Pan and Lee 2016; Faria et al. 2017), and the ACs can be as thermostatically controllable appliances for Direct Load Control (DLC) DR program (Erdinc et al. 2017). Therefore, a SCADA model for investigating user behaviors during DR event, and also validating the implementation of DR programs is very relevant to be considered as part of DR business models.

There is a diverse related research developed in this context. In (Wu et al. 2017), the authors discussed about the energy scheduling challenges in office buildings with the integration of electric vehicles and DRERs. In (Pellegrino et al. 2016) a building management system has been designed and implemented in order to monitor and control the lighting system of office spaces. Reference (Jia et al. 2012) proposed a simulation-based model regarding the resource scheduling for supplying the electrical load in commercial office buildings. In (Mega et al. 2017), an experimental evaluation of a fast DR program for small and medium scale office buildings has been presented. In (Kjaergaard et al. 2016), the authors proposed a system for automated demand response and load control considering the occupant comfort. They also provided a case 
study in a real office building that allows $3 \mathrm{~kW}$ load shedding within comfort limits. Reference (Hao et al. 2017) presented a transactive controlling method for Air Conditioning (AC) of commercial buildings in order to be able to participate in DR programs. All of this related research addresses distinct topics related with the automation devices, controllers, and methods that support the efficient energy management in buildings, namely for the participation in DR programs.

Aiming at presenting a complete solution for the study and demonstration of DR potential available in office buildings from lighting and AC, the present paper brings SCADA-based model which employs several controlling and monitoring means in order to manage the consumption and generation of the building for participating in DR events. In the proposed model, the office building is considered as a member of an aggregator and receives DR programs signal $\mathrm{s}$ in order to fulfil the aggregator goals. In this way, the proposed and implemented system is able to autonomously provide a response of the building to the DR event according to the user's preferences. The proposed model is developed and demonstrated in the present paper for incentive-based DR programs, with focus on an office building with several lighting and AC devices. However, it can easily be adapted to accommodate price-based DR programs and other types of buildings according to the user's preferences. In this system, several real controller components manage the consumption of lighting system and ACs of the building based on the developed optimization model. Such model allows to test and validate the performance of the developed optimization problem in a realistic model and gain the actual measurement results.

After this introductory section, the implemented SCADA model is presented in Section Implemented SCADA model. Section Optimization algorithm represents the proposed optimization model for the SCADA system based on real data. Section Case study explains a case study for the presented model, and its optimization results concerning optimal consumption reduction are shown on Section Results. Finally, the main conclusions of the work will be presented in Section Conclusions.

\section{Implemented SCADA model}

This section presents the implemented SCADA model, by describing the office building plan in sub-section Office building plan and the SCADA installation in sub-section SCADA installation.

\section{Office building plan}

The office building in which the SCADA system has been implemented, belongs to GECAD research centre, located in ISEP/IPP, Porto, Portugal. The building consists of a corridor and 9 offices named N101, N102... N109, that each of which contains typical office equipment, such as personal computers, ACs, and lighting systems. One of these 9 offices (N104) is specified for servers of the building; the SCADA system has specific consideration for this office, since it contains several critical equipment. These offices are equipped with Programmable Logic Controllers (PLCs), several energy meters, different types of sensors, and one main web-based touch screen console to monitor data and control the loads. The 9 offices of the building are divided into 3 zones as it can be seen in Fig. 1. 


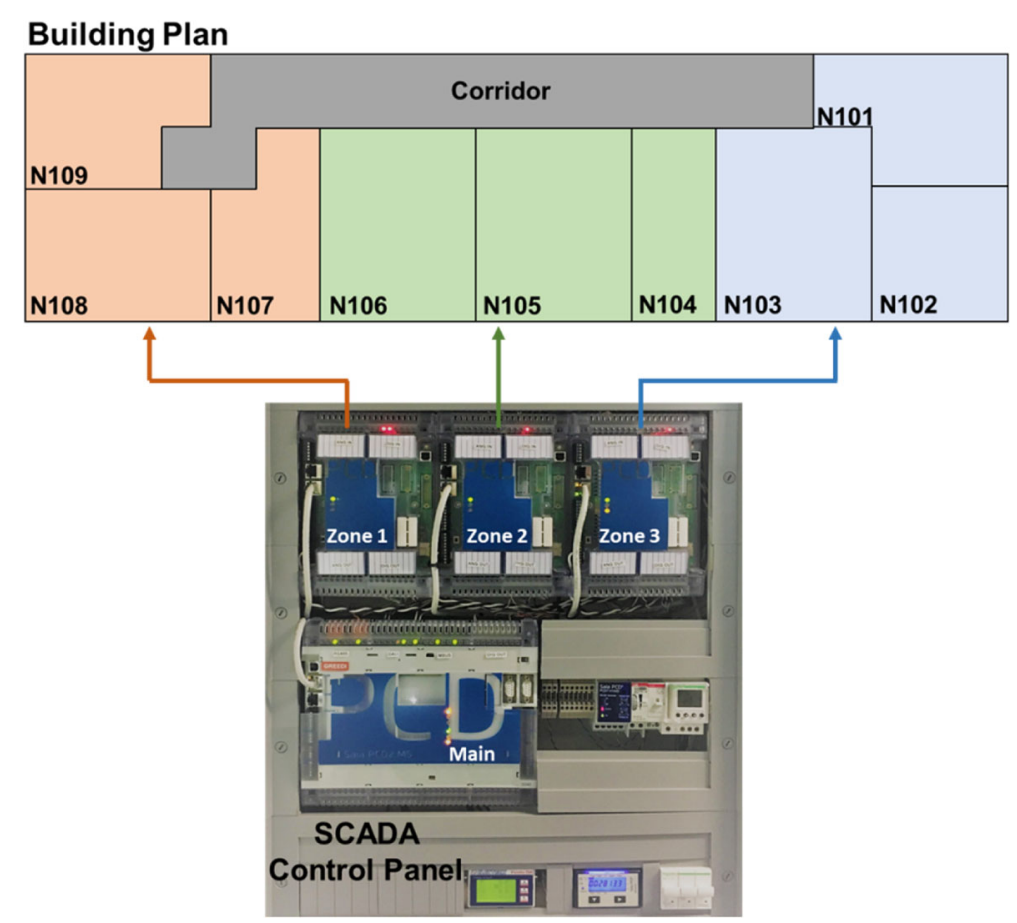

Fig. 1 Plan of the office building and associated zones

According to Fig. 1, the offices N101, N102, and N103 are belong to zone 1, N104 to N106 are dedicated for zone 2, and N107 to N109 are categorized in zone 3. Each zone contains one distributed PLC and one energy meter and a group of sensors.

\section{SCADA installation}

The SCADA system contains PLCs, energy meters, sensors, and a main PLC in the building, to which other distributed PLCs are connected. TCP/IP protocol is used in order to exchange information. The implemented controlling panel of SCADA system is shown on Fig. 2. This SCADA is responsible for monitoring (energy consumption, generation, temperature, humidity, etc.) and controlling of the building. One touch screen console is available in building for monitor and control the data.

All of equipment, including monitoring and controlling devices, are connected to the SCADA system through different communication protocols and Digital/Analog inputs, according to Fig. 3.

The lighting system of this SCADA has fluorescent lights (19 lights in total), and they are fully controllable via Digital Addressable Lighting Interface (DALI). Each light is shown on the touch screen console (Fig. 2) as a button in order to be controlled. By this way, the proposed SCADA system can control the intensity of each light as well as switch them ON/OFF. These kinds of load controlling enable the SCADA system to perform lighting optimization and participate in DR programs.

Regarding the $\mathrm{AC}$ control, there are $10 \mathrm{AC}$ devices available on the building, one in each office and one in corridor. An Arduino ${ }^{\circ}$ (www.arduino.cc) equipped with an Ethernet Shield and an Infrared Light-Emitting Diode (IR LED) have been programmed and installed near to each AC device. Actually, this controlling scenario emulates the 


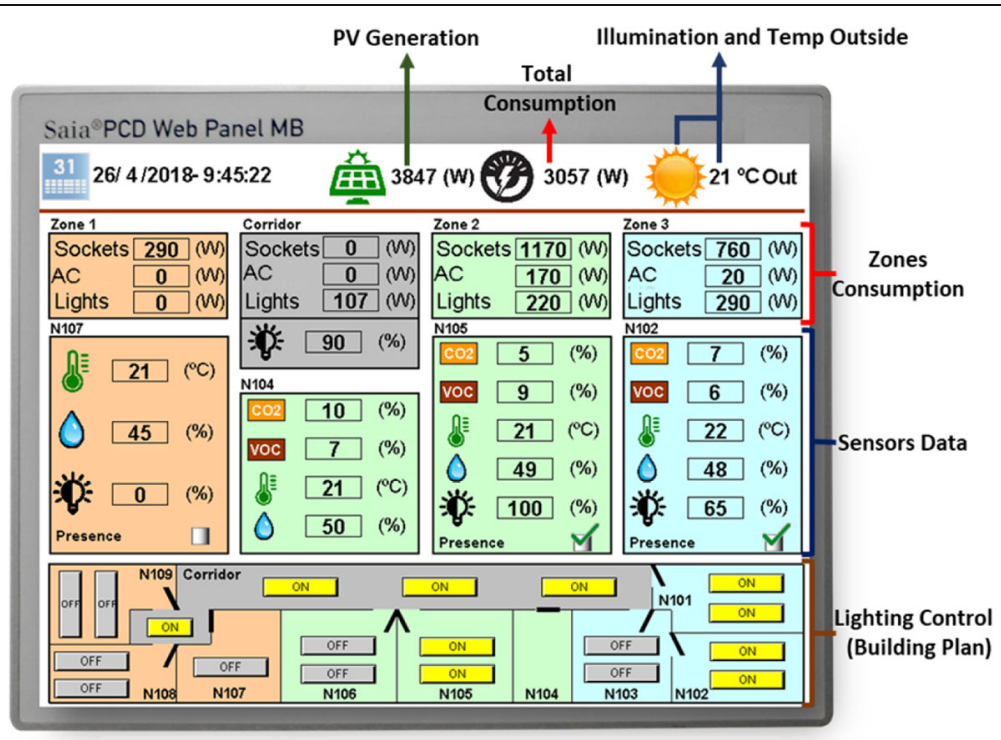

Fig. 2 Designed webpage for monitoring real-time data of SCADA (touch screen console)

remote control of ACs, somehow the SCADA takes decision for each AC and transmits the desired command to each $\mathrm{AC}$ controller $\left(\mathrm{Arduino}^{\circ}\right)$ via Ethernet interface; Arduino controls the AC based on the SCADA decision (turn OFF/ON, and regulating the temperature) and fan for DR participation.

The group of sensors employed in each zone of the SCADA system consist of measuring temperature, humidity, $\mathrm{CO} 2$, air quality (Volatile Organic Compounds - VOC), presence sensor, and illumination intensity sensor. All the sensors (temperature, humidity,

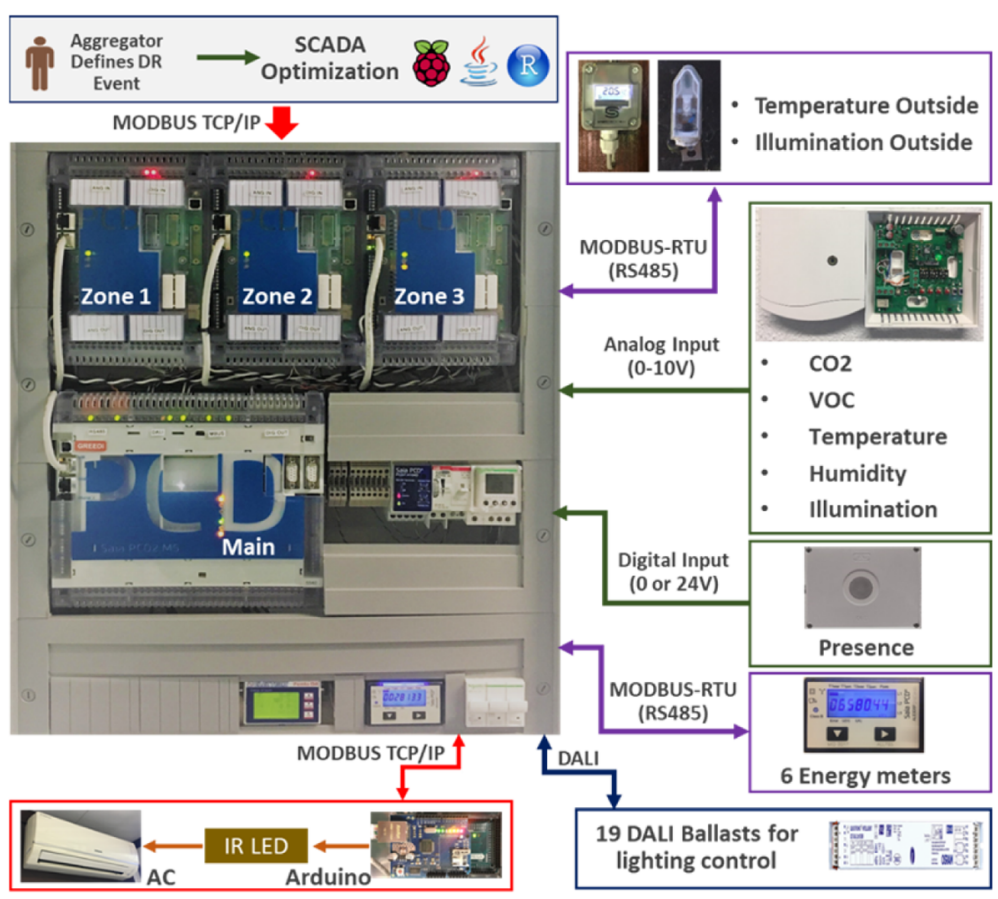

Fig. 3 Implemented SCADA system for an office building controlling and monitoring 
$\mathrm{CO} 2$, air quality, and illumination intensity) are connected to the related PLC in respective zone by $0-10 \mathrm{~V}$ Analog Input.

In this SCADA system, there is a PV system with maximum 7.5 KW capacity. The generated power by PV will supply a part of building consumption, and while there is more generation than the consumption, surplus of generation will be injected to the utility grid. The electricity network of the building has three phases, where phase 1 feeds sockets of the building, phase 2 supplies the ACs, and phase 3 feeds the lighting system. There are six energy meters that measures the consumption and generation of the building. All of these energy meters follow serial communication with MODBUS RS485 protocol. Energy meter \#1 to \#3 measures the consumption of 3 zones and transmit the data to the distributed PLC of each zone, energy meter \#4 measures corridor consumption, meter $\# 5$ measures the total consumption of the building, and energy meter \#6 measures total generation of PV system.

In fact, all of these automation scenarios enable the GECAD office building to have loads reduction and curtailment capabilities in order to participate in real DR case studies and events.

\section{Optimization algorithm}

This section details the algorithm of the optimization model for AC and lighting in GECAD building according to SCADA system introduced before. The overall architecture of the presented optimization problem is illustrated in Fig. 4.

As can be seen in Fig. 4, the optimization algorithm used in this section is started with definition of input data, including total consumption of the building, consumption of the participating devices in the optimization and total required reduction for the optimization algorithm. After that, algorithm starts with the optimization of ACs,

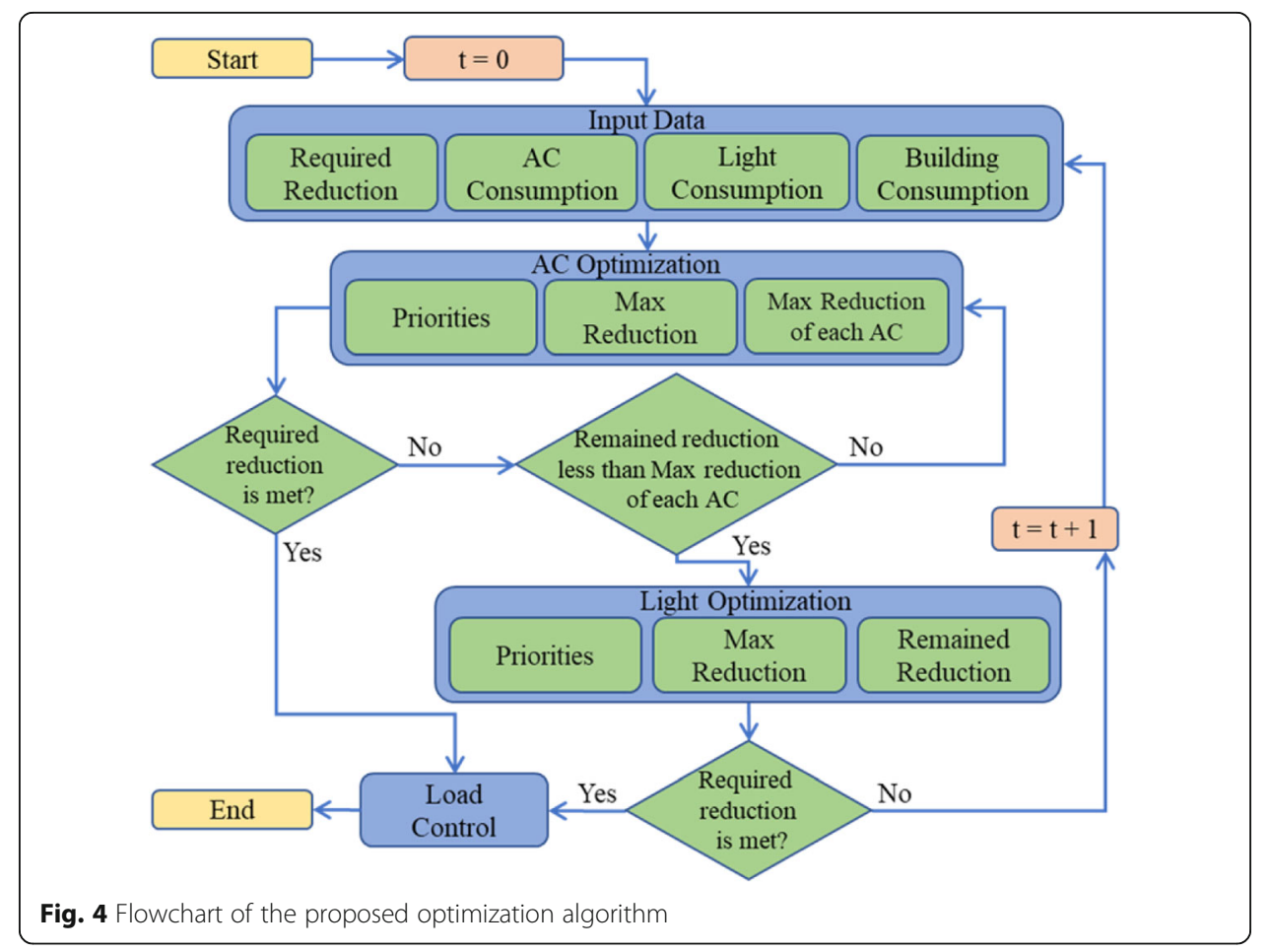


because in this algorithm ACs have priority over the lights to reduce. Then, the algorithm enters to the lights optimization for the remained required reduction if exists. In fact, it depends on the amount of the required reduction of algorithm. If the maximum reduction capacity of $\mathrm{ACs}$ is equal or greater than the required reduction, the algorithm will not enter to the lights optimization process, since the ACs reduction will fulfil the algorithm goal. However, if the required reduction is greater than the maximum reduction capacity of ACs, the algorithm first optimizes the ACs as much as possible and then enters to the lights optimization for the remained required reduction. Therefore, the objective function of optimization problem is demonstrated in Eq. (1):

Minimize

$$
\text { Objective Function }=\sum_{t=1}^{T}\left(\left(\sum_{a=1}^{A} P_{A C . R e d(a, t)} \times W_{A C(a, t)}\right)+\left(\sum_{l=1}^{L} P_{\text {Light.Red }(l, t)} \times W_{\text {Light }(l)}\right)\right)
$$

In this objective function, the weight of priority of the ACs and lights $\left(W_{A C}, W_{L i g h t}\right)$ are defined by each user for the related devices through a webpage available for the office users. The power reductions are determined by the optimization algorithm according to the weight of each lighting and AC device. Also, it is imposed a maximum contribution of each type of device.

The following constraints are proposed for the presented algorithm. Equation (2) shows the required reduction, which should be decreased from the total consumption of the building. Equation (3) indicates that the required reduction for the lighting system depends on the maximum reduction capacity of ACs and the total required reduction of the system. This means that if the total reduction of the system is equal or smaller than the maximum reduction capacity of ACs, there is no need to reduce the lighting consumption.

$$
\begin{aligned}
& \sum_{a=1}^{A} P_{A C . R e d(a, t)}+\sum_{l=1}^{L} P_{\text {Light.Red }(l, t)}=R R_{\text {Total }(t)} \quad \forall 1 \leq t \leq T \\
& R R_{\text {Light }(t)}=R R_{\text {Total }(t)}-P_{A C \cdot R e d(t)}^{M A X} \quad \forall 1 \leq t \leq T
\end{aligned}
$$

Additionally, the maximum reduction capacity of entire ACs and lighting system are represented by Eqs. (4) and (5) respectively. Also, the technical limitations for the maximum reduction of each device are modelled in Eq. (6) for each AC, and Eq. (6) for each light.

$$
\begin{aligned}
& \sum_{a=1}^{A} P_{A C \cdot R e d(a, t)} \leq P_{A C \cdot R e d(t)}^{M A X} ; \forall 1 \leq t \leq T \\
& \sum_{l=1}^{L} P_{\text {Light.Red }(l, t)} \leq P_{\text {Light.Red }(t)}^{M A X} ; \forall 1 \leq t \leq T \\
& P_{A C \cdot \operatorname{Red}(a, t)}^{M I N} \leq P_{A C . R e d(a, t)} \leq P_{A C . R e d(a, t)}^{M A X} ; \forall 1 \leq a \leq A ; \forall 1 \leq t \leq T \\
& P_{\text {Light.Red }(l, t)}^{M I N} \leq P_{\text {Light.Red }(l, t)} \leq P_{\text {Light.Red }(l, t)}^{M A X} ; \forall 1 \leq l \leq L ; \forall 1 \leq t \leq T
\end{aligned}
$$

In sum, the mathematical formulations regarding the optimization of consumption in SCADA system have been represented. In the next section, this model will be used for a case study in order to optimally manage the consumption of building. 


\section{Case study}

In this section, a case study is presented in order to test and validate the proposed system. For this purpose, internal low voltage distribution network of ISEP/IPP university campus in Porto, Portugal, is considered as a network for an aggregator. This university campus microgrid has been adapted from (Silva et al. 2015), and is shown on Fig. 5.

This distribution network consists of underground electrical lines with 21 buses, which a MV/LV transformer located in BUS \#21 connects the microgrid to the main network. There are 21 buildings in the university campus microgrid indicating with yellow color in Fig. 5, somehow each of which is connected to one bus of the network. Therefore, each building can be considered as a member of aggregation network. In fact, the implemented SCADA model is actually located in BUS \#15 of this aggregator network. Figure 6 demonstrates total consumption and generation of GECAD building, which has been monitored by SCADA system. (BUS\#15-GECAD Building).

The profiles shown on Fig. 6 are the real consumption, (including sockets, lights and ACs) and generation data for a typical winter day in 2017, which has been measured by energy meters of SCADA with $1 \mathrm{~s}$ time interval and stored in GECAD database with $10 \mathrm{~s}$ time interval as input data for the optimization algorithm (average of one hour is considered as one period, therefore, 24 periods for $24 \mathrm{~h}$ as shown on Fig. 6). The winter day has been chosen for this case study to validate the performance of the optimization algorithm in the periods that the PV generation is low, and the energy supply relies more on the main grid. In this case study, it is considered that the aggregator meets a drop on its generation resources, and therefore, it provides several DR programs to its customers to keep the network balance, and prevent buying energy from the external supplier. The reason of this lack of generation is assumed as a fault or any other cause in the network.

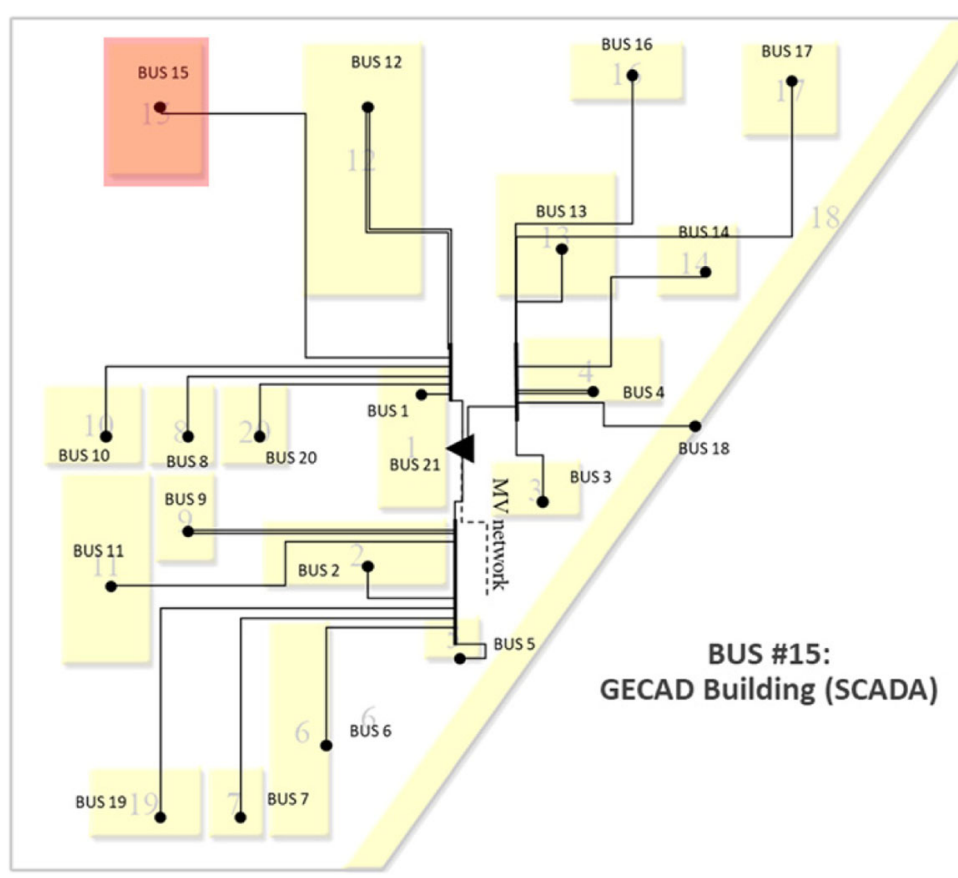

Fig. 5 Low voltage distribution network of university campus considered as an aggregation network 


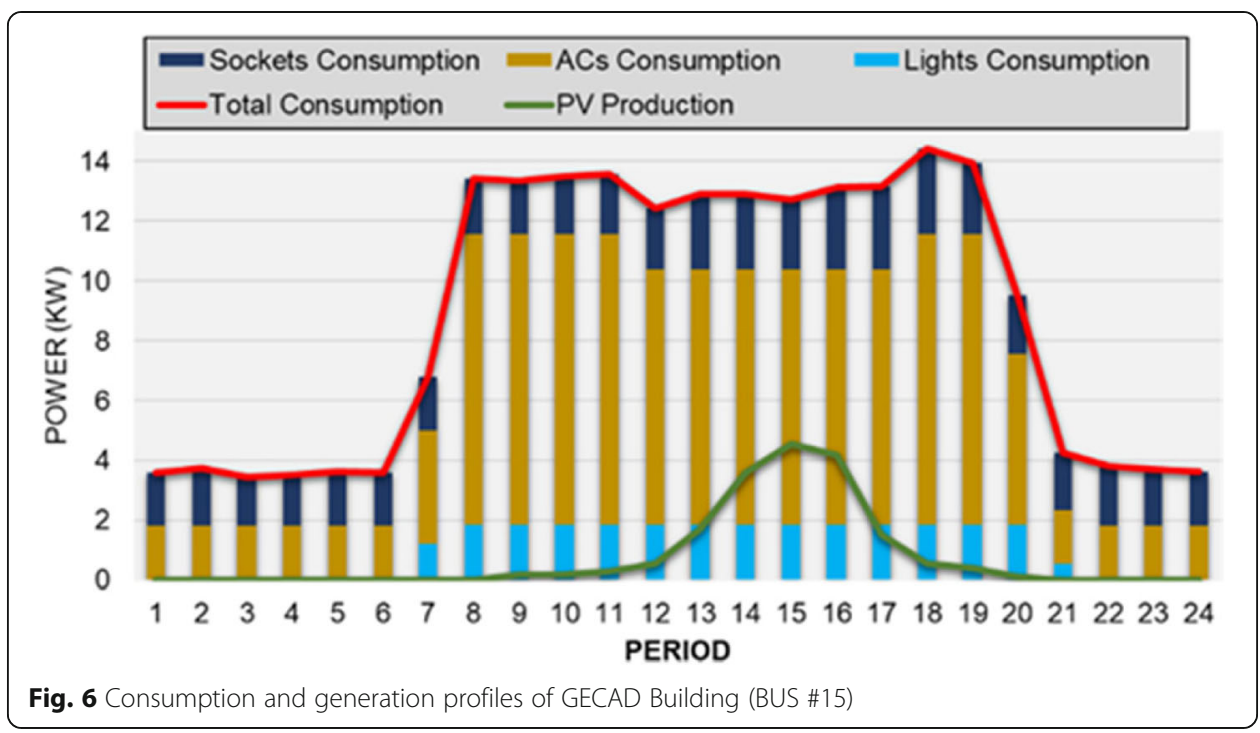

It is considered that customers of the aggregation network have established the DR contracts between aggregator due to participate in DR programs. Therefore, it is considered that GECAD building in BUS \#15 has established a reduction contract with $3.4 \mathrm{~kW}$ reduction capacity from period $\# 9$ to $\# 19$. In this case, it is considered that while the aggregator faced with the lack of generation, it informs GECAD building to reduce $3.4 \mathrm{~kW}$ during period $\# 9$ to $\# 19$. This means, the amount of contracted reduction $(3.4 \mathrm{~kW})$ will be the input (total required reduction) of SCADA optimization algorithm.

\section{Results}

This section illustrates the results of optimization problem proposed in this paper. This algorithm has been solved via "IpsolveAPI" tools of RStudio (www.rstudio.com). As the first result, Fig. 7 demonstrates the optimal consumption reduction of GECAD building, which is the result of optimization problem.

As you can see in Fig. 7, SCADA optimization process is started from period \#9 and is finished on \#19. In these periods, as it mentioned before, the total required reduction of system is set on $3.4 \mathrm{~kW}$, in order to reduce the consumption based on the priorities of devices available on SCADA. By this way, the system first starts to optimize the consumption of ACs (low priorities devices will be interrupted first), and then, if the ACs reduction is not sufficient, the algorithm starts to optimize lighting system based on their priorities. Moreover, Fig. 8 demonstrates the detailed amount of reduction of lights and ACs in each zone.

During the optimization process, different ACs are reduced in different periods since various priorities are defined by each user for each period. As it was mentioned, the priorities of ACs and lights are defined by each user for the associated devices through a webpage available for the office users. Therefore, the optimization algorithm will reduce or interrupt the devices with low priorities first. As can be seen in Fig. 8 - (a), the optimization reduced two ACs in each zone, which are the ones with low priority, in order to attain the total reduction of system. However, there are some periods that AC reduction is not adequate, therefore the optimization will entire to the light reduction 


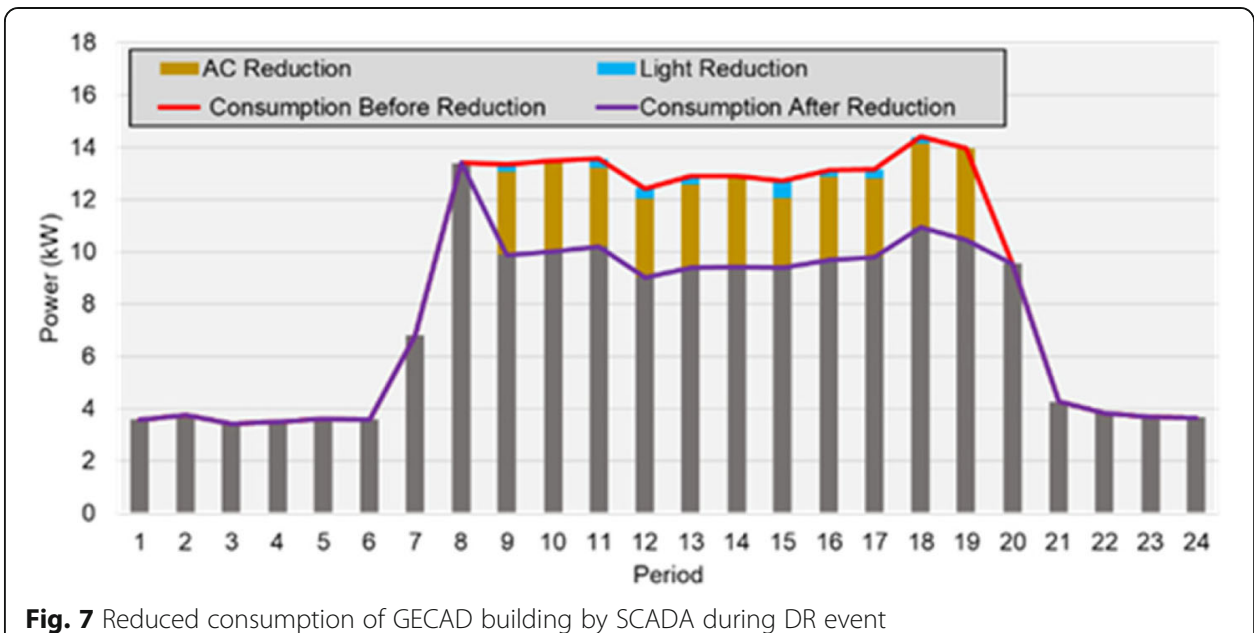

as Fig. 8 - (b) shows. Similar to ACs, the lights with low priority is reduced first. Then, the system reduced the high priority lights. Also, there are some periods during optimization, namely period \#10, \#14, and \#19, the AC reduction attains to around $3.4 \mathrm{~kW}$, and therefore, there is no reduction in the lighting system.

Beside the optimization results, the illumination and temperature in each office are important, in order to do not disturb user preferences. Therefore, intensity of illumination as well as temperature of one office in each zone are illustrated by Fig. 9 .

In order to demonstrate the conditions of the illumination and temperature in offices during the DR events, office $\mathrm{N} 102$ has been selected from zone 1 to survey, as

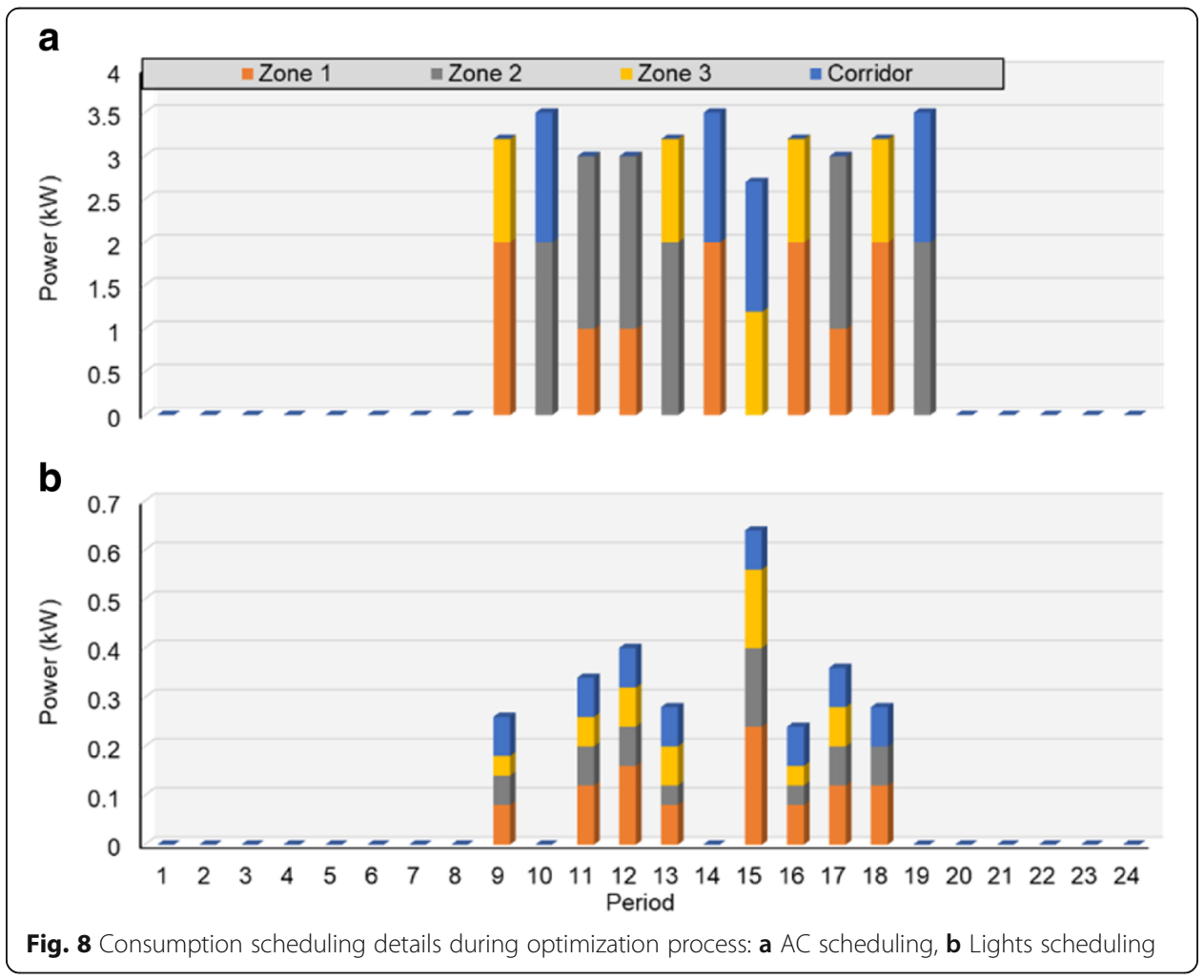


illustrated in Fig. 9 - (a). Also, office N105 in zone 2 and office N107 in zone 3 have been selected as demonstrated in Fig. 9 - (b) and Fig. 9 - (c) respectively. The data shown on Fig. 9 are the real data measured and monitored by SCADA system, which shows the periods that office users start working (around period \#7-8), and leave the work (around period \#20-21). Also, since the selected day for case study was a winter day, the users utilized the AC for heating the office, and therefore, the temperature increased during the working hours (shown by a line in Fig. 9). Furthermore, as it is clear in Fig. 9, by reducing consumption lights in each office, the illumination intensity is not much affected, and probably the user will not feel the reduction. This is true also for the AC reduction, which means the optimization process managed the ACs somehow that the user will be dissatisfaction for temperature variations.

While further control actions are not implemented, the users are able to go to the same webpage where the priorities are defined and change the actual priority of a device by changing the current operation mode. The system automatically selects other device to reduce the consumption.

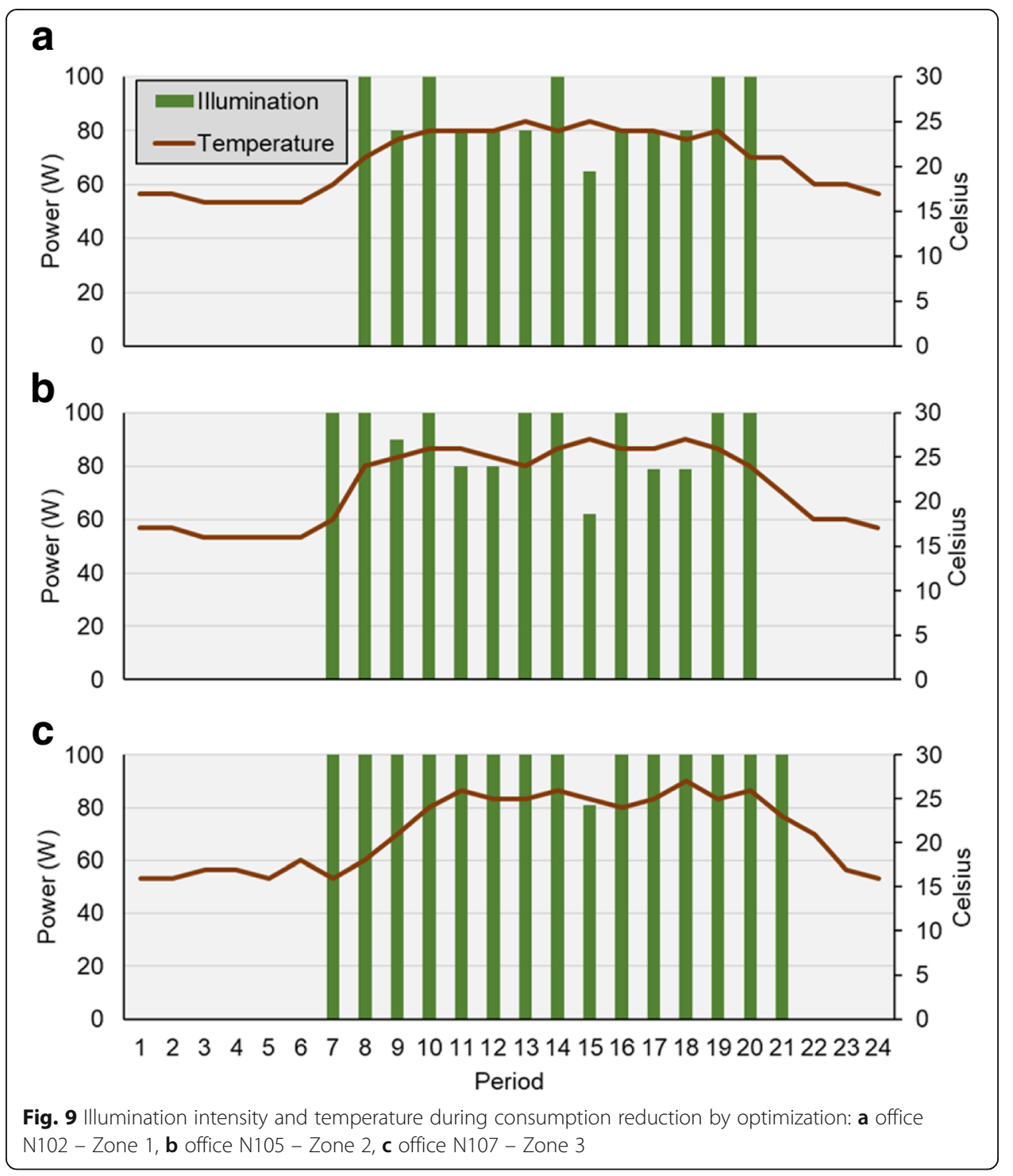




\section{Conclusions}

In this paper, a real SCADA model implemented for an office building has been proposed in order be able to make decision and participate in demand response events. Several distributed based programmable logic controllers managed the consumption and generation of building. Furthermore, several types of sensors as well as energy meters are used in this SCADA model for monitoring the condition of the building. Moreover, the SCADA model controls lighting system via digital addressable lighting interface, and air conditioners via ethernet interface with MODBUS protocol.

An optimization algorithm for consumption reduction of this office building based on the priorities of components have been proposed. This optimization problem focuses on the air conditioners and lighting system of the implemented SCADA system. The optimization algorithm utilizes several real data from SCADA, and starts to manage the consumption based on these real data. For the case study, distribution network of a university campus, where actually the implemented SCADA model is located, has been used as an aggregator model, in order to provide demand response programs.

The results of case study prove the importance of SCADA system for executing demand response program, and also demonstrate that with small reduction in the lighting system and air conditioners, the customers would be able to participate in demand response program while their preferences are not much affected.

\section{Nomenclature}

\section{Parameters}

$P_{A C . R e d}$ Power reduction of AC

$P_{\text {Light. Red }}$ Power reduction of light

$P_{A C . R e d}^{M A X}$ Maximum reduction of AC

$P_{\text {Light.Red }}^{M A X}$ Maximum reduction of lights

$P_{A C . R e d}^{M I N}$ Minimum reduction of AC

$P_{\text {Light.Red }}^{\text {MIN }}$ Minimum reduction of lights

$W_{A C}$ Weight of priority of AC

$W_{\text {Light }}$ Weight of priority of light

$R R_{\text {Total }}$ Total required reduction

$R R_{\text {Light }}$ Required reduction of lights

\section{Indexes}

$T$ Number of time periods

A Maximum number of ACs

$L$ Maximum number of lights

Funding

The present work has been developed under the EUREKA - ITEA2 Project FUSE-IT (ITEA-13023), Project GREEDI

(ANI|P202017822) co-funded by Portugal 2020 "Fundo Europeu de Desenvolvimento Regional" (FEDER) through PO Cl, and UID/EEA/00760/2013. 


\section{Authors' contributions}

MK implemented the optimization model and analyzed the results. OA contributed in the SCADA model. ZV participated in the design of the study. PF conceived of the study, and participated in its design and coordination and helped to draft the manuscript. All authors read and approved the final manuscript.

\section{Ethics approval and consent to participate}

Not applicable.

\section{Consent for publication}

Not applicable.

\section{Competing interests}

The authors declare that they have no competing interests.

\section{Publisher's Note}

Springer Nature remains neutral with regard to jurisdictional claims in published maps and institutional affiliations.

Received: 9 January 2018 Accepted: 11 June 2018

Published online: 24 August 2018

\section{References}

Abrishambaf O, Faria P, Gomes L, Spínola J, Vale Z, Corchado J (2017) Implementation of a real-time microgrid simulation platform based on centralized and distributed management. Energies 10(6):806

Abrishambaf O, Ghazvini M, Gomes L, Faria P, Vale Z, Corchado J (2016) Application of a Home Energy Management System for Incentive-Based Demand Response Program Implementation. Paper presented at 27th International Workshop On Database And Expert Systems Applications (DEXA), Porto, Portugal, 5-8 Sept. 2016

Erdinc O, Tascikaraoglu A, Paterakis N, Catalao J (2017) An energy credit based incentive mechanism for the direct load control of residential HVAC systems incorporation in day-ahead planning. Paper presented at 2017 IEEE Manchester Powertech, Manchester, UK, 18-22 June 2017

Faria P, Pinto A, Vale Z, Khorram M (2017) Lighting Consumption Optimization using Fish School Search Algorithm. Paper presented at 2017 IEEE Symposium on Computational Intelligence Applications in Smart Grid (IEEE CIASG'17), Hawaii, USA, 27-1 Nov/Dec 2017

Faria P, Soares J, Vale Z, Morais H, Sousa T (2013) Modified particle swarm optimization applied to integrated demand response and DG resources scheduling. IEEE Trans Smart Grid 4(1):606-616

Faria P, Spinola J, Vale Z (2016) Aggregation and remuneration of electricity consumers and producers for the definition of demand-response programs. IEEE Trans Ind Inf 12(3):952-961

Faria P, Vale Z (2011) Demand response in electrical energy supply: an optimal real time pricing approach. Energy 36(8):5374-5384

Fotouhi Ghazvini M, Soares J, Abrishambaf O, Castro R, Vale Z (2017) Demand response implementation in smart households. Energ Buildings 143:129-148

Hao H, Corbin C, Kalsi K, Pratt R (2017) Transactive control of commercial buildings for demand response. IEEE Trans Power Sys 32(1):774-783

Hasan M, Mouftah H (2016) Optimal trust system placement in smart grid SCADA networks. IEEE Access 4:2907-2919

Hu J, Cao J, Yong T, Guerrero J, Chen M, Li Y (2017) Demand response load following of source and load systems. IEEE Trans Control Syst Technol 25(5):1586-1598

Jia Q, Shen J, Xu Z, Guan X (2012) Simulation-based policy improvement for energy Management in Commercial Office Buildings. IEEE Trans Smart Grid 3(4):2211-2223

Kjaergaard, M., Arendt, K., Clausen, A., Johansen, A., Jradi, M., Jorgensen, B. et al. (2016). Demand response in commercial buildings with an Assessable impact on occupant comfort. Paper presented at IEEE International Conference On Smart Grid Communications (Smartgridcomm), Sydney, NSW, Australia, 6-9 Nov. 2016

Kwon S, Ntaimo L, Gautam N (2017) Optimal day-ahead power procurement with renewable energy and demand response. IEEE Trans Power Syst 32(5):3924-3933

Lujano-Rojas J, Dufo-Lopez R, Bernal-Agustin J, Catalao J (2017) Optimizing daily operation of battery energy storage systems under real-time pricing schemes. IEEE Trans Smart Grid 8(1):316-330

Mega, T., Kitagami, S., Kawawaki, S., Kushiro, N. (2017). Experimental Evaluation of a Fast Demand Response System for Small/Medium-Scale Office Buildings. Paper presented at 31St International Conference On Advanced Information Networking And Applications Workshops (WAINA), Taipei, Taiwan, 27-29 Mar. 2017

Pan X, Lee B (2016) An Approach of Reinforcement Learning Based Lighting Control for Demand Response. Paper presented at PCIM Europe 2016; International Exhibition and Conference for Power Electronics, Intelligent Motion, Renew Energy and Energy Management, Nuremberg, Germany, 10-12 May 2016

Parvania M, Fotuhi-Firuzabad M, Shahidehpour M (2013) Optimal demand response aggregation in wholesale electricity markets. IEEE Trans Smart Grid 4(4):1957-1965

Paterakis N, Erdinç O, Catalão J (2017) An overview of demand response: key-elements and international experience. Renew Sust Energ Rev 69:871-891

Pellegrino A, Lo Verso V, Blaso L, Acquaviva A, Patti E, Osello A (2016) Lighting control and monitoring for energy efficiency: a case study focused on the interoperability of building management systems. IEEE Trans Ind App 52(3):2627-2637

Ruelens F, Claessens B, Vandael S, De Schutter B, Babuska R, Belmans R (2017) Residential demand response of thermostatically controlled loads using batch reinforcement learning. IEEE Trans Smart Grid 8(5):2149-2159 
Silva M, Fernandes F, Morais H, Ramos S, Vale Z (2015) Hour-ahead energy resource management in university campus microgrid. Paper presented at IEEE Eindhoven Powertech, Eindhoven, Netherlands, 29 June-2 July 2015

Wang F, Xu H, Xu T, Li K, Shafie-khah M, Catalão J (2017) The values of market-based demand response on improving power system reliability under extreme circumstances. Appl Energy 193:220-231

Wu D, Zeng H, Lu C, Boulet B (2017) Two-stage energy Management for Office Buildings with Workplace EV charging and renewable energy. IEEE Trans Transp Electrification 3(1):225-237

\section{Submit your manuscript to a SpringerOpen ${ }^{\circ}$} journal and benefit from:

- Convenient online submission

- Rigorous peer review

- Open access: articles freely available online

- High visibility within the field

- Retaining the copyright to your article

Submit your next manuscript at $\gg$ springeropen.com 